\title{
Levantamento do desempenho logístico das PMEs da indústria de bens de capital: uma análise comparativa
}

\author{
Danilo Hisano Barbosa ${ }^{a *}$, Marcel Andreotti Musetti ${ }^{\mathrm{b}}$ \\ a*hisano@sc.usp.br, EESC-USP, Brasil \\ bmusetti@sc.usp.br, EESC-USP, Brasil
}

\begin{abstract}
Resumo
Este artigo apresenta os resultados de um levantamento sobre o desempenho logístico das PMEs da indústria de bens de capital. 0 desempenho logístico foi avaliado com base no grau de concordância das empresas frente às afirmações acerca das dimensões do desempenho logístico identificadas na literatura. Os resultados indicaram uma concordância maior das empresas frente a essas afirmações. Nas análises comparativas, foi observado que as médias de desempenho das PMEs pesquisadas distanciaram-se significativamente do padrão de excelência em logística. Adicionalmente, foram constatadas diferenças entre as médias de desempenho logístico nas comparações por segmento, porte e origem de capital das PMEs. Entre as implicações práticas e teóricas, destacam-se a necessidade de criação de parcerias entre as PMEs nacionais e estrangeiras e a oportunidade de se sistematizar as práticas de excelência em logística do setor, visando a sua transferência para as empresas de menor desempenho logístico.
\end{abstract}

Palavras-chave

Logística. Excelência logística. Desempenho logístico. Indústria de bens de capital.

\section{Introdução}

0 desempenho logístico tem se tornado uma poderosa fonte de vantagem competitiva nos últimos anos, pois as empresas passaram a enxergar a logística não mais como simples fonte de redução de custos, mas também como fonte de melhoria aos produtos e serviços oferecidos aos clientes (MCDUFFIE et al., 2001; LAPIDE, 2006).

Nesse sentido, o alcance de tal desempenho tem sido objeto de estudo de diversos trabalhos de pesquisa. Essas pesquisas mostram que a excelência em logística depende basicamente de fatores relacionados à eficiência dos estoques e nível de serviço ao cliente, sendo estes: mudanças frequentes no giro e níveis de estoques, diminuição nas alocações de estoques, a existência de estratégias logísticas específicas para lidar com diferentes clientes e a flexibilidade no atendimento a pedidos especiais (FAWCETT; CLINTON, 1996).
Em relação à indústria brasileira de bens de capital, esta pode ser caracterizada como difusora de novas tecnologias para outros setores da economia e representa um importante setor da produção industrial do país. Segundo informações da Pesquisa Industrial de lnovação Tecnológica (PINTEC) realizada pelo IBGE em 2008 (INSTITUTO..., 2008), houve um ligeiro aumento no número de empresas que atribuem importância alta à aquisição de máquinas e equipamentos no país, passando dos $76,6 \% \mathrm{em}$ 2000 para 77,7\% em 2008.

No Brasil, as pesquisas sobre essa indústria normalmente focam em estudos econômicos, tais como indicadores de produção física, grau de inovação e na caracterização e classificação de seus segmentos ou subsetores. Outras pesquisas relatam a importância da logística nesse setor, pois se trata da movimentação de materiais de grande porte e alto valor agregado, o que provoca um alto custo logístico para as empresas 
(BRANDÃO, 2003). Apesar dessa problemática, pouco se conhece sobre o contexto logístico das pequenas e médias empresas (PMEs) desse setor no país.

Sabe-se que as PMEs geram resultados significativos para a economia paulista e nacional. De acordo com os dados do Sebrae (2011), no Brasil existem 5,1 milhões de empresas. Desse total, 98\% são micro e pequenas empresas. Esse grupo apresenta faturamento correspondente a 20\% do PIB nacional. No estado de São Paulo, o faturamento é de $28 \%$ do total da atividade empresarial paulista, respondendo por mais de $2 / 3$ dos empregos. A partir desses dados, constata-se a importância dessas empresas como fonte geradora de empregos e promotora do desenvolvimento socioeconômico.

Diante dessa importância, o presente artigo destaca os resultados de uma pesquisa de levantamento (survey), que é parte integrante da Rede de Pesquisa Instituto Fábrica do Milênio 11 (IFM 11), conduzida com o objetivo de identificar e analisar o desempenho logístico de empresas do setor de bens de capital. 0 recorte analítico dado neste artigo recaiu sobre o desempenho logístico das PMEs da indústria de bens de capital (IBK), onde foram realizadas análises comparativas por porte, origem e segmento e com os valores de referência existentes na literatura para as empresas normais e líderes em logística.

Primeiramente, selecionaram-se os três segmentos considerados como os mais representativos do setor seguindo critérios de produção física, impacto na cadeia exportadora e importância econômica. Foram utilizados os dados de 2008 da Associação Brasileira da Indústria de Máquinas e Equipamentos (ABIMAQ). Tais segmentos correspondem primeiramente ao de bens de capitais agrícolas, seguido pelos bens de capitais para fins industriais não seriados, principalmente aqueles voltados à extração mineral e bens de capitais para transportes.

Após a seleção desses segmentos, foi consultada a base de dados da Abimaq, sendo identificado um total aproximado de 264 empresas dos três segmentos. Todas essas empresas foram contatadas inicialmente por telefone, mas muitas não aceitaram participar da pesquisa, sendo o principal motivo alegado o sigilo associado à área. Conseguiram-se com os telefonemas 182 contatos (e-mails) de gestores, que foram utilizados posteriormente para o envio dos questionários via correio eletrônico (mail survey). Dos 182 questionários enviados, 41 retornaram devidamente preenchidos. $\mathrm{Na}$ análise preliminar deles, foram eliminados aqueles respondidos por empresas de grande porte, pelo foco do estudo ser as PMEs do setor. Dessa forma, apenas 33 foram reportados à análise, totalizando um índice de retorno de aproximadamente 18\%. Esse índice de retorno está coerente com o de estudos empíricos sobre desempenho logístico ou nível de excelência logística de empresas manufatureiras realizadas em outros países (CLOSS; XU, 2000; FAWCETT; CLINTON, 1997; MOLLENKOPF; DAPIRAN, 2005).

Inicialmente é apresentada uma breve revisão bibliográfica contemplando a caracterização da indústria de bens de capital e uma descrição da literatura especializada sobre desempenho logístico. Em seguida, apresenta-se o método de pesquisa, os resultados obtidos e as análises. Por fim, são discutidas as conclusões gerais da pesquisa.

\section{Revisão da literatura}

\subsection{A indústria de bens de capital}

A importância da IBK é inconteste e pode ser vista através do seu grau de influência na dinâmica econômica de um país (SOUZA, 2004), do seu perfil difusor e transmissor de novas tecnologias para outros setores econômicos (BAARK, 1991), de sua potencialidade na geração de mão de obra qualificada no mercado doméstico (WEISE, 2000). No Brasil, a sua representatividade pode ser vista também em seu potencial de gerar inovação. A aquisição de bens de capital é considerada pela Pesquisa Industrial de Inovação Tecnológica (2008) como a atividade de inovação de maior importância no país, com 77,7\% das empresas destacando-a como relevante para desenvolver suas inovações.

A indústria de bens de capital é composta por seis segmentos de acordo com a finalidade de seus produtos, sendo estes: agrícolas, transportes, construção civil, fins industriais seriados e não seriados, energia elétrica e de uso misto (INSTITUT0..., 2008). Para fins deste artigo, focou-se nos três segmentos mais representativos da IBK brasileira, segundo critérios como produção física, impacto na cadeia exportadora e importância estratégica para a economia nacional mensurada pela dinamicidade de sua inovação tecnológica ou pelo seu impacto em cadeias produtivas fundamentais. Esses subsetores correspondem ao de bens de capitais agrícolas, seguido pelos bens de capitais para fins industriais não seriados, principalmente aqueles voltados à extração mineral e bens de capitais para transportes. 0 processo de classificação desses subsetores será explicado em detalhes na seção de metodologia.

No que tange às características gerais do setor, estas compreendem principalmente a sua heterogeneidade e seu aspecto difusor de tecnologias. A heterogeneidade pode ser vista na abrangência dos produtos do setor, estendendo-se desde machados até refrigeradores da linha branca (INSTITUTO..., 2008). Já em relação ao 
seu aspecto difusor de tecnologias, o setor ocupa um papel central no desenvolvimento e difusão das mesmas, aumentando os níveis de produtividade e capacidade tecnológica em setores manufatureiros e não manufatureiros (BAARK, 1991).

Entretanto, é nas características das vendas do setor que reside a importância da logística, pois tratam-se de vendas envolvendo produtos de alto valor agregado (preço) e baixo volume (quantidade), customizados e frequentemente únicos para determinada necessidade, 0 que faz do custo logístico um dos principais problemas enfrentados pelas empresas do setor (BRANDÃO, 2003). Além do custo, outra importante preocupação dos gestores logísticos do setor relaciona-se com o serviço ao cliente e o gerenciamento de ativos, sendo estes os principais motivadores para a realização de projetos de melhoria e mudança na área de logística dessas empresas.

Esse contexto revela que as dimensões primárias do desempenho logístico, estoques e nível de serviço ao cliente exercem grande influência na competitividade das empresas do setor, fazendo-se necessária a escolha de um instrumento que avalie e caracterize o desempenho logístico dessas empresas, assunto tratado na próxima seção.

\subsection{Desempenho logístico}

0 desempenho logístico é entendido nesse artigo dentro de suas principais dimensões, estando a primeira relacionada a custo e eficiência, nas quais se incluem os estoques, e a segunda ao nível de serviço ao cliente (CLOSS; SWINK; NAIR, 2005; FAWCETT; CLINTON, 1996; GUNASEKARAN; PATEL; TIRTIROGLU, 2001; LAPIDE, 2006; LAMBERT; POHLEN, 2001; ROSENWEIG; ROTH; DEAN, 2003; SCHRAMM-KLEIN; MORSCHETT, 2006).

Essas dimensões ganharam importância, pois as empresas passaram a enxergar a logística não mais como uma simples fonte de redução de estoques e custos, mas também como fonte de melhoria aos produtos e serviços oferecidos aos clientes. Nesse sentido, repensar as competências logísticas pode resultar em ideias criativas para melhorar simultaneamente essas duas dimensões (GLOBAL..., 1995; MCDUFFIE et al., 2001). Além disso, essa importância torna-se ainda mais representativa no Brasil, visto que o alto custo logístico e a variabilidade do serviço prestado ao cliente sempre foram os principais desafios enfrentados pelos gestores logísticos brasileiros (BOWERSOX; CLOSS, 1997). Para os autores, a demanda e interesse em se atingir a excelência logística entre as empresas brasileiras é significativa, sendo uma oportunidade para gestores e acadêmicos em geral.
São escassas as publicações que tratam da logística no contexto das PMEs. Entre as que abordam especificamente o desempenho logístico nessas empresas, estão a de Halley e Guilhon (1997) e mais recentemente as de Koh et al. (2007) e Töyli et al. (2008). Para Halley e Guilhon (1997), em função da crescente conscientização sobre a integração organizacional, a logística vem aos poucos se tornando o principal assunto das empresas de pequeno e médio porte e sua gestão continua sendo centralizada nos sócios-proprietários dessas empresas, que têm um alto controle dessa atividade. Para os autores, a logística desempenha basicamente duas funções nas PMEs. A primeira consiste na função de adaptação, auxiliando a empresa a se adequar às mudanças do ambiente externo, e a segunda refere-se à função de integração dos vários níveis organizacionais que a logística desempenha.

Já Töyli et al. (2008) desenvolveram um estudo, cujo enfoque foi o desempenho logístico das PMEs, avaliado por meio de suas dimensões. Os resultados dessa pesquisa indicaram que os níveis de serviços aos clientes e a eficiência em custos estão positivamente correlacionados nessas empresas, embora as que tenham alto nível de serviço ao cliente tendem a terem ao mesmo tempo baixos custos logísticos. Tais descobertas revelam que a logística está despertando maior atenção entre as PMEs, e seria oportuno que essas empresas obtivessem vantagem competitiva focando nas duas dimensões primárias do desempenho logístico. Já a pesquisa de Koh et al. (2007) contribui com o corpo de conhecimento existente ao fornecer novos dados e perspectivas empíricas sobre a relação entre as práticas de gestão da cadeia de suprimentos e o desempenho logístico das PMEs operantes na Turquia.

Outra forma de se avaliar o desempenho logístico se dá através de modelos de excelência em logística. Essa avaliação pode se pautar numa competência logística específica (CLOSS; XU, 2000) ou no desempenho logístico como um todo, envolvendo todas as suas dimensões (FAWCETT; CLINTON, 1997). Closs e Xu (2000) utilizaram-se de uma das dimensões do modelo de excelência logística desenvolvido pelo Global Logistics Research Team (1995), que trata das tecnologias de informação logísticas, para avaliar essa competência em empresas americanas, europeias e asiáticas. Já Fawcett e Clinton (1997) utilizaram-se das dimensões de seu modelo de excelência criado em 1996 para avaliar e comparar o nível de desenvolvimento logístico de empresas de três diferentes países.

Já nesse artigo, o recorte analítico é dado na sétima dimensão do modelo de Fawcett e Clinton (1996), denominada de desempenho logístico e que, segundo os autores, pode ser utilizada para avaliar o desempenho logístico de empresas de 
manufatura, sendo composta pelos seguintes itens: mudanças frequentes no giro e níveis de estoques, diminuição na alocação de estoques pela empresa, existência de estratégias logísticas específicas para lidar com diferentes consumidores e a flexibilidade no atendimento a pedidos especiais de clientes. Tal estrutura formulada em 1996 foi validada pelos autores em outro estudo, realizado em 1997, envolvendo 1.900 empresas. 0 Alpha de Cronbach para este instrumento foi relativamente alto, 0.76 - isto indica que os cinco itens possuem consistência interna e, portanto, podem ser utilizados para se avaliar o desempenho logístico de empresas de manufatura (FAWCETT; CLINTON, 1996). 0 instrumento pode ser visto na Tabela 1.

A lógica do instrumento, segundo os autores, é a seguinte: o desempenho logístico é baseado no princípio de que os níveis e giros de estoques provêm uma medida de custo e eficiência e, embora sejam importantes, a logística tem sido cobrada cada vez mais em oferecer satisfação e serviço ao cliente. Assim, a habilidade em atender pedidos especiais de clientes e a existência de estratégias logísticas específicas para lidar com diferentes consumidores tornaram-se igualmente importantes componentes do serviço logístico.

Deste modo, observa-se na Tabela 1 que os três primeiros itens do instrumento de Fawcett e Clinton (1996) relacionam-se com a dimensão de estoques e os dois últimos com a de nível de serviço ao cliente, corroborando com a visão de outros autores da literatura especializada no que concerne às duas dimensões primárias do desempenho logístico, conforme já destacado no início dessa seção (CLOSS; SWINK; NAIR, 2005; GUNASEKARAN; PATEL; TIRTIROGLU, 2001; LAPIDE, 2006; LAMBERT; POHLEN, 2001; ROSENWEIG; ROTH; DEAN, 2003; SCHRAMM-KLEIN; MORSCHETT, 2006).

\section{Método de pesquisa}

A estratégia de pesquisa considerada mais adequada ao objetivo do trabalho é o levantamento ou survey, que em muitos casos é utilizado para fins descritivos, objetivando-se entender a relevância de um fenômeno e como ele se configura no âmbito de uma população (FORZA, 2002). Para o autor, pensar através de fatos descritivos pode fornecer ideias valorosas para a construção e refinamento de teorias. Para efeito deste survey, ressalta-se que o processo de amostragem de pesquisa implicou na exclusão de uma parte das empresas filiadas à Abimaq (por estas não pertencerem aos subsetores selecionados para o estudo).

0 processo de seleção dos subsetores foi o seguinte. Primeiramente, buscou-se encontrar um índice denominado de Índice de Priorização do Setor (IPS), sendo este calculado por meio da multiplicação de critérios tais como: produção física, impacto na cadeia exportadora brasileira e importância estratégica para a economia nacional (mensurada pela dinamicidade de sua inovação tecnológica ou pelo seu impacto em cadeias produtivas fundamentais). Após a obtenção desse índice, foi realizada uma análise de pareto, na qual identificou-se o conjunto de setores com maior IPS. Os resultados apontaram que um conjunto pequeno de segmentos respondia pelo maior valor de IPS e ao realizar um corte de IPS maior ou igual a 6 chegou-se a um conjunto de segmentos em ordem de grandeza suficiente para se obter a classificação. Tais subsetores corresponderam primeiramente ao de bens de capitais agrícolas, seguido pelos subsetores, bens de capitais para fins industriais não seriados, principalmente aqueles voltados à extração mineral, e bens de capitais para transportes.

Dessa forma, a população total, com potencial para fazer parte da amostra pesquisada, era estimada em 264 empresas. Todas essas empresas foram contatadas por telefone pela equipe de pesquisadores com o objetivo de se obter contatos (e-mails) dos responsáveis pela área de logística ou diretores industriais. Foi obtido um total de 182 e-mails e realizadas 3 ondas de envios dos questionários, nas quais puderam ser aproveitados apenas 41 , que retornaram devidamente preenchidos.

Devido o foco do trabalho nas PMEs do setor, foram selecionadas somente as respostas relativas a essas empresas, totalizando 33 respostas, que foram reportadas à análise. 0 índice de retorno de aproximadamente $18 \%$ é coerente ao de pesquisas sobre avaliação do desempenho logístico ou nível

Tabela 1. Construto de Fawcett e Clinton (1996).

\begin{tabular}{|c|c|c|c|c|c|}
\hline \multirow{2}{*}{$\begin{array}{c}\text { Desempenho logístico } \\
1.0 \text { giro de estoque na empresa tem aumentado nos últimos cinco anos? }\end{array}$} & \multicolumn{2}{|c|}{$\begin{array}{c}\text { Discordo } \\
\text { totalmente }\end{array}$} & \multirow{2}{*}{$\longleftrightarrow$} & \multicolumn{2}{|c|}{$\begin{array}{l}\text { Concordo } \\
\text { totalmente }\end{array}$} \\
\hline & 1 & 2 & & 4 & 5 \\
\hline 2. 0 nível de estoque médio da empresa diminuiu se comparado a cinco anos atrás? & 1 & 2 & 3 & 4 & 5 \\
\hline 3. A empresa tem estoques alocados em menos locais hoje se comparado a cinco anos atrás? & 1 & 2 & 3 & 4 & 5 \\
\hline 4. A empresa é flexível no atendimento a pedidos especiais de clientes? & 1 & 2 & 3 & 4 & 5 \\
\hline 5. A empresa possui estratégias de logística específicas para lidar com diferentes consumidores? & 1 & 2 & 3 & 4 & 5 \\
\hline
\end{tabular}


de excelência logística de empresas de manufatura realizadas em outros países (FAWCETT; CLINTON, 1996, 1997; MOLLENKOPF; DAPIRAN, 2005).

Em relação ao instrumento de coleta de dados, destaca-se o questionário semiestruturado com questões predominantemente fechadas, elaborado com base no construto de Fawcett e Clinton (1996), construto este já validado por uma survey com 1.900 empresas em 1997. A versão final do questionário acabou sendo composta por dois principais blocos de questões, estando o primeiro relacionado à caracterização da empresa e o segundo ao escopo da pesquisa, que consiste em avaliar o nível de desempenho logístico das empresas.

Para a análise, optou-se pelo agrupamento dos dados em tabelas de frequência e a construção de gráficos de barras, além da aplicação de um teste estatístico não paramétrico para a comparação de proporções, o teste binomial. Além disso, os dados foram cruzados, permitindo identificar e apontar as principais descobertas e resultados da pesquisa.

\section{Resultados}

\subsection{Amostra da pesquisa}

Com relação ao capital, 5 (15,15\%) do total de empresas pesquisadas são de origem estrangeira (multinacionais) e $28(84,85 \%)$ são nacionais. Outra informação interessante é que 79\% das empresas não pertencem a nenhum grupo empresarial.

No que se refere à faixa de faturamento médio anual, a maioria das empresas $(81,8 \%)$ encontrava-se na primeira faixa, a de até 100 milhões de reais e apenas $9,1 \%$ na maior faixa de faturamento, acima de 1 bilhão de reais. Já no que se refere à exportação, pode-se dizer que $75,8 \%$ das empresas pesquisadas têm menos de $20 \%$ do faturamento oriundo de exportação. As Tabelas 2 e 3 detalham essas duas informações.

Quanto ao porte de empresas conforme o seu número de funcionários, destaca-se que $9,1 \%$ podem ser consideradas microempresas, $36,4 \%$ pequenas e $54,5 \%$ médias empresas. Tal classificação baseou-se no critério do Sebrae (2011) para o porte de empresas segundo o seu número de funcionários. Para a instituição, as PMEs englobam as micro, pequenas e médias empresas e possuem até 500 funcionários. As microempresas são formadas por até 19 funcionários, as pequenas entre 20 e 99 e as médias empresas são aquelas que empregam entre 100 e 499 funcionários. A respectiva caracterização pode ser vista na Figura 1. Já a distribuição das PMEs por segmentos da IBK é apresentada na Figura 2.
Tabela 2. Distribuição das empresas respondentes por faixas de faturamento anual.

\begin{tabular}{cc}
\hline $\begin{array}{c}\text { Faixas de faturamento anual } \\
\text { (em R\$ milhões) }\end{array}$ & $\begin{array}{c}\text { \% das empresas } \\
\text { pesquisadas }\end{array}$ \\
\hline $0-99$ & 81,8 \\
$100-499$ & 9,1 \\
$500-999$ & - \\
Acima de 1 bilhão & 9,1 \\
Total & 100 \\
\hline
\end{tabular}

Tabela 3. Distribuição das empresas respondentes quanto à exportação.

\begin{tabular}{cc}
\hline $\begin{array}{c}\text { \% do faturamento anual exportado } \\
\text { (em R\$ milhões) }\end{array}$ & $\begin{array}{c}\text { \% das empresas } \\
\text { pesquisadas }\end{array}$ \\
\hline Não exporta & 9,1 \\
Até 20 & 75,8 \\
$21-50$ & 12,1 \\
$51-70$ & 3,0 \\
Acima de 70\% & - \\
Total & 100,0 \\
\hline
\end{tabular}

Constata-se com base nos dados apresentados que as PMEs caracterizam-se, em sua maioria, como empresas nacionais de médio porte não pertencentes a grupos empresariais, com faixa de faturamento de até 100 milhões de reais e moderado perfil exportador.

\subsection{Desempenho logístico}

A Tabela 4 apresenta as médias de respostas das empresas pesquisadas para cada item do desempenho logístico analisado. A menor média de desempenho logístico ocorreu no quarto item, que trata da flexibilidade da empresa no atendimento a pedidos especiais de clientes.

Distribuindo as respostas dessas empresas na escala de cinco pontos ( 1 - discordo totalmente a 5 - concordo totalmente), verificou-se uma tendência maior de concordância do que de discordância das empresas em todos os itens do desempenho logístico analisados, conforme mostra a Figura 3. Os dados indicam que aproximadamente $64 \%$ das empresas concordam que os giros de estoques aumentaram nos últimos cinco anos, contra $21 \%$ que discordam.

No segundo item analisado, 57,6\% das empresas concordam com a afirmação de que o nível de estoque médio diminuiu se comparado a cinco anos atrás contra 30,3\% que discordam dessa afirmação. No terceiro item, as diferenças de respostas foram mais discrepantes, 66,7\% que concordam contra 15,1\% que discordam. Do mesmo modo, na dimensão de serviço ao cliente, as empresas tenderam a respostas de concordância, sendo esta mais intensa no último 


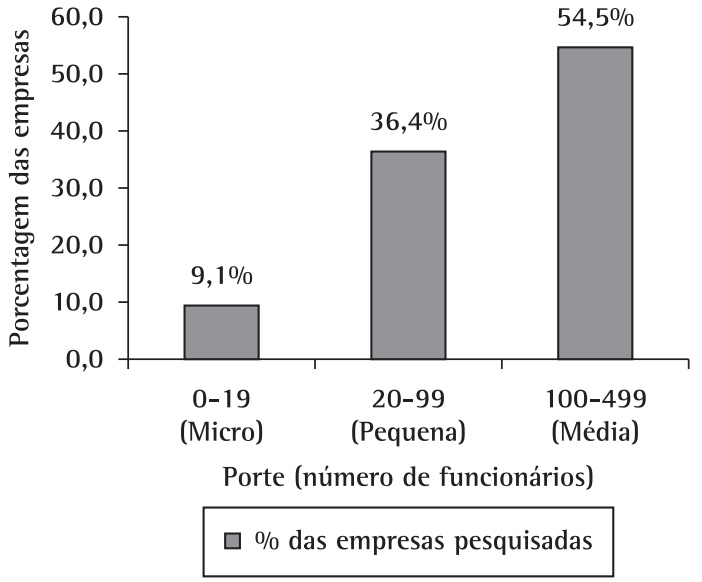

Figura 1. Distribuição das PMEs respondentes por porte.

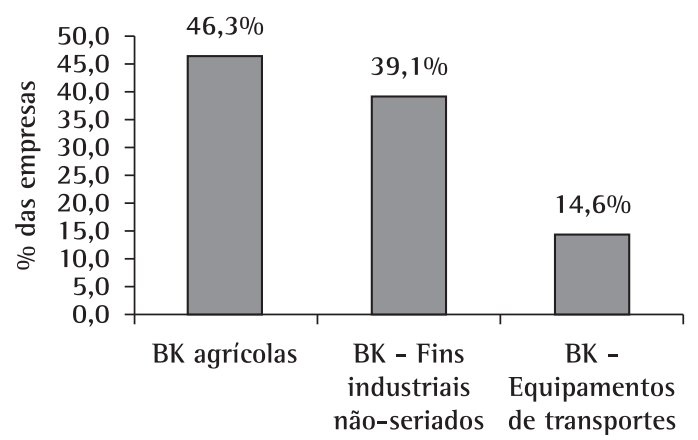

Segmentos da Indústria de Bens de Capital (IBK)

$\square \%$ das empresas pesquisadas

Figura 2. Distribuição das PMEs respondentes por segmentos da IBK.

Tabela 4. Médias de desempenho logístico das empresas pesquisadas.

\begin{tabular}{lc}
\hline \multicolumn{1}{c}{ Desempenho logístico } & Média \\
\hline $\begin{array}{l}\text { O giro de estoque na empresa tem aumentado nos } \\
\text { últimos cinco anos? }\end{array}$ & 3,61 \\
$\begin{array}{l}\text { O nível de estoque médio da empresa diminuiu se } \\
\text { comparado a cinco anos atrás? }\end{array}$ & 3,30 \\
$\begin{array}{l}\text { A empresa tem estoques alocados em menos locais } \\
\text { hoje se comparado a cinco anos atrás? }\end{array}$ & 3,76 \\
$\begin{array}{l}\text { A empresa é flexível no atendimento a pedidos } \\
\text { especiais de clientes? }\end{array}$ & 2,91 \\
$\begin{array}{l}\text { A empresa possui estratégias de logística específicas } \\
\text { para lidar com diferentes consumidores? }\end{array}$ & 3,64 \\
Composto pelo desempenho logístico & 17,22 \\
\hline
\end{tabular}

item analisado, com $66,7 \%$ das empresas concordando com a afirmação.

Para comprovar estatisticamente a significância das diferenças entre essas proporções foi utilizado o teste binomial, que consiste em um teste estatístico não paramétrico para a comparação de proporções (BOWER, 1996). Esse teste requer duas alternativas ou categorias de respostas diferentes, tais como: sim ou não, masculino ou feminino, sucesso ou insucesso, concorda ou discorda, etc. Dessa forma, os pontos 4 e 5 e 1 e 2 da escala Likert foram agrupados em duas categorias de respostas (concorda ou discorda) e as comparações ocorreram na proporção das empresas que concordam ou discordam das afirmações, sendo desconsideradas as respostas neutras, por não terem força de valor no que se refere à posição das empresas. Para essa análise foi utilizado o software estatístico SPSS 10.0.

Os resultados indicaram diferenças significativas nas proporções das duas categorias de resposta (concordam ou discordam), indicando uma concordância majoritária das empresas respondentes face aos itens 1,3 e 5 , que apresentaram $\mathrm{p}$-valores $<0,05$. Já no segundo e quarto item do desempenho logístico analisado, os resultados não mostraram diferenças significativas na distribuição de proporções entre as duas categorias, 0 que indica que as posições dos participantes quanto a esta questão se encontraram relativamente divididas. A Tabela 5 apresenta os resultados do teste binomial.

\subsubsection{Análises comparativas}

Ao comparar as médias com os valores de referência de Fawcett e Clinton (1996), constatou-se que as médias ficaram abaixo do padrão das empresas líderes e próximas ao desempenho de empresas normais, principalmente no terceiro e quinto item, onde o desempenho foi ligeiramente superior ao padrão das normais, conforme Figura 4.

Já na comparação entre o desempenho logístico das PMEs nacionais e estrangeiras (Figura 5), foi constatada a maior média das subsidiárias estrangeiras. Tal fato pode ser explicado em função dessas empresas já trazerem as competências, conhecimentos e experiências logísticas adquiridas no exterior e até mesmo por seguirem a conduta da empresa matriz.

$\mathrm{Na}$ comparação por segmentos da lBK, destacou-se o desempenho dos bens de capital - equipamentos de transportes, que apresentou a maior média frente aos demais, 3,75. Já os segmentos de bens de capital agrícolas e para fins industriais não seriados, apresentaram médias de desempenho de 3,1 e 3,68 respectivamente, conforme pode ser visto na Figura 6.

Para realizar a comparação por porte de empresa, as PMEs foram divididas nos subgrupos que a constituem, sendo estas as micro, pequenas e médias empresas. As médias de desempenho foram maiores nas empresas de pequeno e médio porte $(3,47)$ em contraste com as microempresas, que apresentaram média de 3,2, conforme destaca a Figura 7.

A comparação do desempenho das PMEs com o valor de referência de Fawcett e Clinton (1996) 


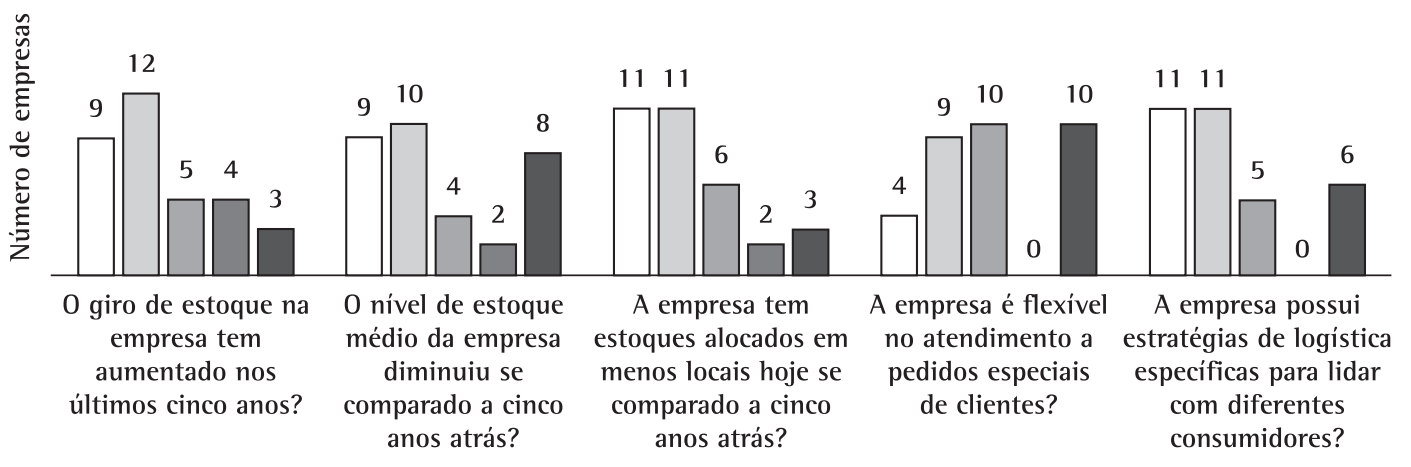

Questões sobre o desempenho logístico das empresas

$\square$ Concordo totalmente $\square$ Concordo $\square$ Neutro $\square$ Discordo $\square$ Discordo totalmente

Figura 3. Distribuição das respostas das PMEs na escala de cinco pontos (padrão likert).

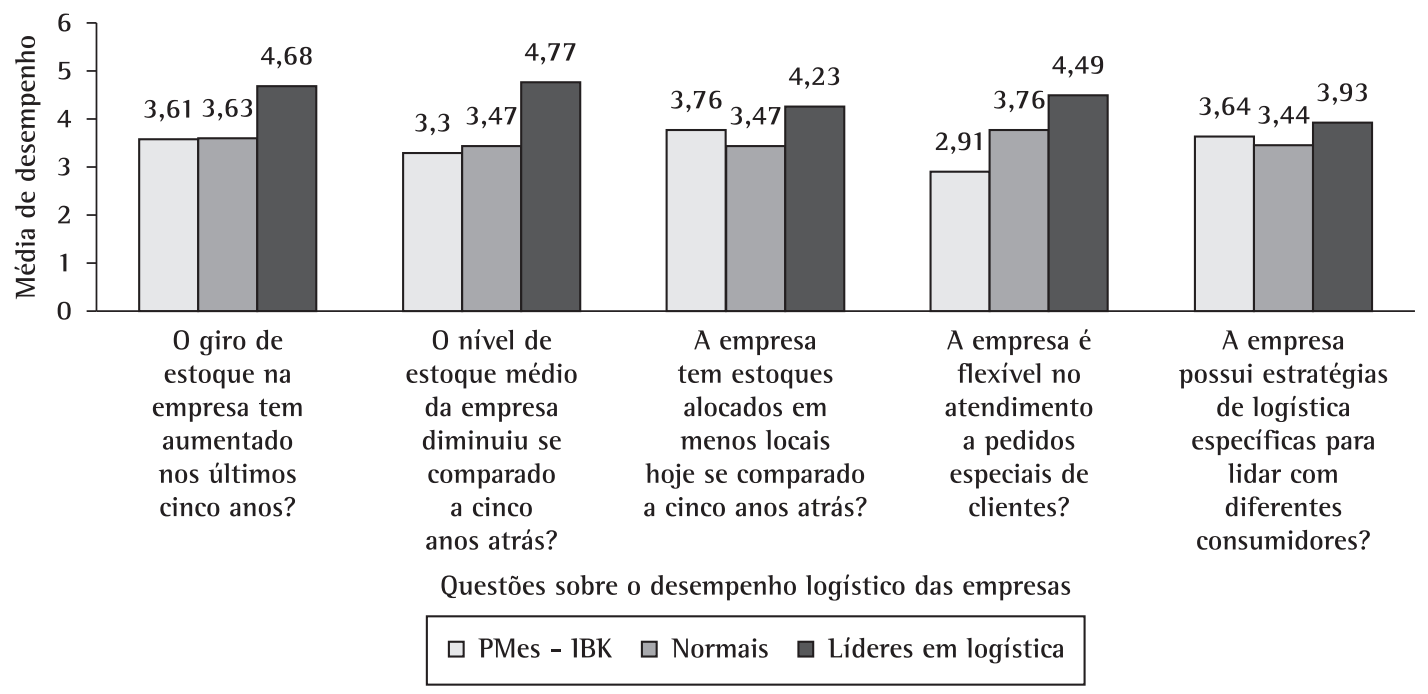

Figura 4. Comparações com o desempenho logístico de empresas normais e líderes em logística (Valores de referência de Fawcett e Clinton (1996)).

Tabela 5. Resultados do teste binomial.

\begin{tabular}{lrrr}
\hline \multicolumn{1}{c}{ Desempenho logístico } & $\begin{array}{c}\text { Concordam } \\
\text { (n) }\end{array}$ & $\begin{array}{c}\text { Discordam } \\
\text { (n) }\end{array}$ & $\begin{array}{c}\text { p-valor } \\
\text { (teste binomial) }\end{array}$ \\
\hline 0 giro de estoque na empresa tem aumentado nos últimos cinco anos? & 21 & 7 & 0,013 \\
0 nível de estoque médio da empresa diminuiu se comparado a cinco anos atrás? & 19 & 10 & 0,136 \\
A empresa tem estoques alocados em menos locais hoje se comparado a cinco anos atrás? & 22 & 5 & 0,002 \\
A empresa é flexível no atendimento a pedidos especiais de clientes? & 13 & 10 & 0,678 \\
A empresa possui estratégias de logística específicas para lidar com diferentes consumidores? & 22 & 6 & 0,004 \\
\hline
\end{tabular}

revelou um momento de transição dessas empresas para padrões mais elevados de desempenho logístico, próximos ao de empresas líderes. Um dos destaques é a existência de estratégias logísticas específicas para lidar com diferentes consumidores. Para Fawcett e Clinton (1996), essa competência é essencial ao alcance da excelência em logística, pois permite que as empresas respondam de forma mais eficaz a pedidos especiais de clientes, e que uma lista de serviços diferenciados seja criada para atender a suas diferentes necessidades. 0 desenvolvimento dessa competência pode ser explicado, de certa forma, pelas iniciativas realizadas pelas PMEs nos últimos anos, entre elas o estabelecimento de relacionamento de parcerias com clientes regulares e o investimento em adoção de práticas gerenciais 


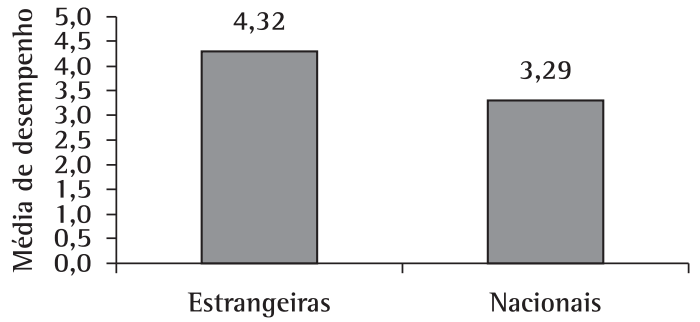

Origem de capital

Figura 5. Comparações das médias de desempenho logístico por origem de capital das PMEs.

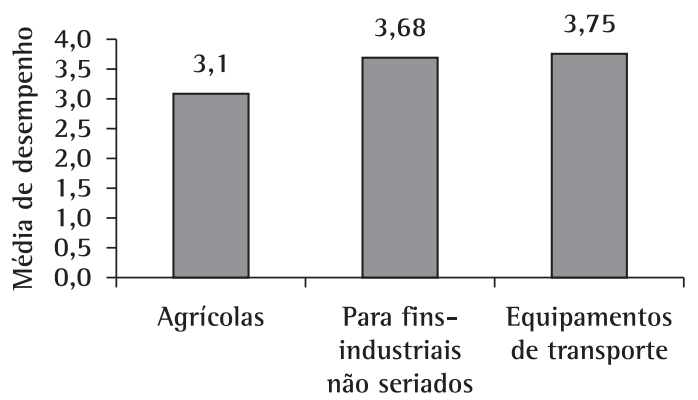

Segmentos da Indústria de Bens de Capital (IBK)

Figura 6. Comparações das médias de desempenho logístico por segmentos da IBK das PMEs.

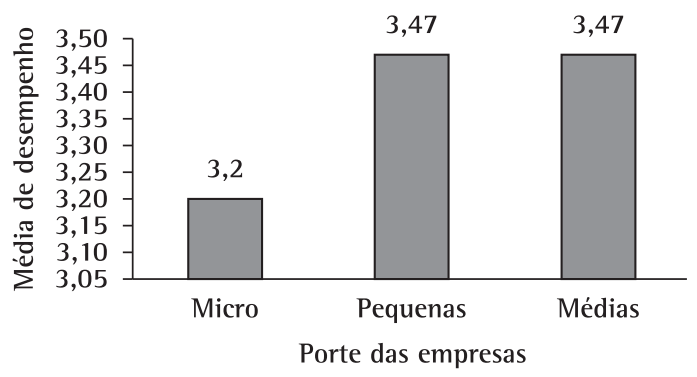

Figura 7. Comparações das médias de desempenho logístico por estratos das PMEs.

voltadas à melhoria do serviço (GlLMORE; CARSON; GRANT, 2001).

Já o desempenho referente à gestão de estoques corrobora as evidências da literatura de que as PMEs vêm sofrendo com relação a essa gestão. Para Rajeev (2008), esses problemas ocorrem devido à baixa importância atribuída a previsão, ordenação aleatória de materiais, baixos níveis de treinamento e desenvolvimento, inadequada utilização de recursos computacionais, baixa importância conferida ao processo de compras e redução do tempo de processamento (lead-time). 0 estudo confirmou a necessidade de uma mudança imediata por parte das PMEs em relação às suas abordagens de gestão de estoques.
Em relação ao cenário brasileiro, observa-se que diversas iniciativas governamentais vêm apoiando o crescimento das PMEs, entre elas o Finame, uma linha de crédito voltada para incentivar a produção, inovação e a aquisição de máquinas e equipamentos (BANCO..., 2011), e o Sebrae, que oferece empréstimos às micro e pequenas empresas brasileiras (SEBRAE, 2011). Espera-se que iniciativas como essas continuem e que estudos futuros abordem a logística dentro do contexto das PMEs.

\section{Conclusões e trabalhos futuros}

Os resultados do teste binomial revelaram uma proporção maior de concordância do que discordância das empresas respondentes em três dos cinco itens do desempenho logístico analisados. 0 primeiro relaciona-se com o giro de estoques, nas quais as empresas concordam que este indicador tem aumentado nos últimos cinco anos, diferindo significativamente das empresas que discordam dessa afirmação (p-valor de 0,013$)$. 0 segundo se refere à alocação de estoques, nas quais foram constatadas diferenças significativas entre as proporções das empresas que concordam ou discordam dessa afirmação (p-valor de 0,002), com concordância majoritária das empresas. 0 último item nas quais foram constatadas diferenças significativas entre essas proporções está relacionado com a existência de estratégias de logística específicas para lidar com diferentes consumidores (p-valor de 0,004), na qual a maioria das empresas concordaram com essa afirmação.

No que se refere às análises comparativas, os resultados revelaram diferenças entre o desempenho logístico das PMEs pesquisadas com o de empresas líderes em logística segundo valores de referência de Fawcett e Clinton (1996), sendo tal desempenho mais próximo dos valores de referência dos autores para as empresas normais. Tal fato pode indicar um momento de transição dessas empresas, já que se tratam de empresas emergentes. Além disso, com as médias de desempenho encontradas no estudo foi possível constatar que o instrumento dos autores permanece coerente na avaliação do desempenho logístico de empresas de manufatura mesmo após anos de sua publicação, não devendo ser considerado ultrapassado.

Dentro do setor estudado, as diferenças entre as médias das empresas nacionais e estrangeiras podem ser explicadas pelo fato de as estrangeiras trazerem consigo as competências, habilidades e conhecimentos logísticos adquiridos no exterior e até mesmo por seguirem a conduta da empresa matriz.

A identificação do nível de desempenho logístico das empresas pesquisadas oferece uma oportunidade 
de identificar as principais tendências logísticas, além de servir também como um diagnóstico das principais carências ou pontos de melhorias. É sugerido que pesquisas futuras abordem outros setores envolvendo as demais dimensões do desempenho logístico não abordadas neste artigo tais como o papel da orientação estratégica e mecanismos de integração e gestão de alianças e o suporte da medição de desempenho e sistemas de informação.

Já a principal contribuição da pesquisa reside na sua originalidade ao estudar e comparar o desempenho logístico das PMEs brasileiras da IBK com padrões de referências internacionais, tendo um papel importante no diagnóstico do grau de desenvolvimento logístico das empresas nacionais, trazendo implicações tanto para gestores como futuras pesquisas.

Entre as principais implicações dos resultados dessa pesquisa para os gestores logísticos, destacam-se:

- 0 desafio de promoverem a criação de parcerias entre as empresas nacionais e as subsidiárias estrangeiras instaladas no país para a transferência de competências, habilidades e experiências logísticas melhorando o desempenho das empresas PMEs nacionais e, consequentemente, a competitividade do setor de bens de capital como um todo. 0 mesmo se aplica para empresas de diferentes portes;

- Mesmo não sendo constatadas diferenças significantes nas médias de desempenho entre os segmentos da IBK, permanece um desafio aos gestores logísticos das PMEs, o de continuarem aperfeiçoando suas práticas logísticas, de forma a alcançar um nível de desempenho superior, já que as análises apontaram um distanciamento relativamente grande desse desempenho quando comparado com o padrão de excelência.

Entre as implicações para futuras pesquisas, destacam-se:

- A necessidade de se sistematizar as práticas de excelência em logística do setor: grupos de pesquisas deveriam explorar com maior profundidade as práticas logísticas de empresas de excelência do setor, sistematizando-as e propondo a aplicação e adaptação destas em empresas de menor desempenho. Essas pesquisas, além de serem importantes do ponto de vista acadêmico, podem trazer outras implicações práticas, como melhoria do desempenho logístico e competitividade do setor e maior conscientização dessa realidade entre os gestores logísticos do país;

- Abordar outros segmentos da IBK: pesquisas futuras poderiam analisar o desempenho logístico nos segmentos da lBK não abordados nesse estudo, como os de bens de capital para o setor de construção, energia elétrica e de uso misto;

- Estudo aprofundado das dimensões primárias do desempenho logístico: a perspectiva de considerar inventário e serviço ao cliente como as principais dimensões do desempenho logístico (CLOSS; SWINK; NAIR, 2005; FAWCETT; CLINTON, 1996; GUNASEKARAN; PATEL; TIRTIROGLU, 2001; LAPIDE, 2006; LAMBERT; POHLEN, 2001; ROSENWEIG; ROTH; DEAN, 2003; SCHRAMM-KLEIN; MORSCHETT, 2006) mostrou ser muito útil nesse estudo, e utilizar-se dessa abordagem em um futuro estudo de caso tornaria possível, por exemplo, avaliar como a combinação ou até mesmo os trade-offs entre essas duas dimensões poderiam impactar de diferentes formas no desempenho logístico.

Entre as principais limitações da pesquisa, a primeira reside no seu pequeno número amostral, que ocorreu em função da resistência das empresas em participar da pesquisa, impedindo a realização de outras análises estatísticas que poderiam ser úteis em apontar novas descobertas. Adicionalmente, destaca-se que o estudo com a qual foram comparados os dados dessa pesquisa possui amostras distintas e foi publicado anos atrás. Para inferir maiores conclusões, estudos futuros poderiam superar essa limitação pela condução de pesquisas em outras regiões brasileiras, com o mesmo planejamento amostral e questionário. Esses estudos seriam importantes para o entendimento de como as questões contextuais podem influenciar no desempenho logístico de empresas de manufatura.

\section{Referências}

ASSOCIAÇÃO NACIONAL DE FABRICANTES DE CERÂMICA PARA REVESTIMENTO - ANFACER. Portal Datamaq. Disponivel em: <http://www.datamaq.org.br>. Acesso em: 20 abr. 2008.

ALEM, A. C.; PESSOA, R. M. 0 setor de bens de capital e o desenvolvimento econômico: quais são os desafios? BNDES Setorial, n. 22, p. 71-88, 2005.

BAARK, E. The Accumulation of technology: capital goods production in developing countries revisited. World Development, v. 19, n. 7, p. 903-914, 1991. http://dx.doi. org/10.1016/0305-750X(91)90141-4

BANCO NACIONAL DE DESENVOLVIMENTO ECONÔMICO E SOCIAL - BNDES. Finame - Maquinas e Equipamentos. Disponível em: http://www.bndes.gov.br. Acesso em: 02 jun. 2011.

BOWER, J. A. Statistics for food science 11l: sensory evaluation data. Part B - discrimination tests. Nutrition \& Food Science, v. 96, n. 2, 1996. http://dx.doi.org/10.1108/00346659610108975

BOWERSOX, D. J.; CLOSS, D. J. Brazilian logistics: A time for transition. Gestão \& Producão, v. 4, n. 2, p. 130-139, 1997. http://dx.doi.org/10.1590/S0104-530X1997000200002

BRANDÃO, F. Sinopse Competitiva da Indústria de Máquinas Agrícolas. In: CONGRESSO SUL BRASILEIRO DA INDÚSTRIA AUTOMOTIVA, 2., 2003, Porto Alegre. Anais... Porto Alegre: Instituto Gaúcho de Estudos Automotivos, Porto Alegre, 2003.

CLOSS, D. J.; SWINK, M.; NAIR, A. The role of information connectivity in making flexible logistics programs successful. International Journal of Physical Distribution and Logistics Management, v. 35, n. 4, p. 258-77, 2005. http://dx.doi.org/10.1108/09600030510599922 
CLOSS, D. J.; XU, K. Logistics information technology practice in manufacturing and merchandising firms: an international benchmarking study versus world class logistics firms. International. Journal of Physical Distribution and Logistics Management, v. 30, n. 10, p. 869-86, 2000. http://dx.doi. org/10.1108/09600030010351264

FAWCETT, S. E.; CLINTON, S. R. Enhancing logistics performance to improve the competitiveness of manufacturing organizations. Production and Inventory Management, v. 37, n. 1, p. 40-6, 1996.

FAWCETT, S. E.; CLINTON, S. R. Enhancing logistics to improve the competitiveness of manufacturing organizations: a triad perspective. Transportation Journal, v. 37, n. 1, p. 18-28, 1997.

FORZA, C. Survey research in operations management: a process-based perspective. International Journal of Operations and Production Management, v. 22, n. 2, p. 152-94, 2002. http://dx.doi.org/10.1108/01443570210414310

GILMORE, A.; CARSON, D.; GRANT, K. SME Marketing in Practice. Marketing Intelligence \& Planning, v. 19, n. 1, p. 6-11, 2001. http://dx.doi.org/10.1108/02634500110363583

GLOBAL LOGISTICS RESEARCH TEAM AT MICHIGAN STATE UNIVERSITY. World Class Logistics: The Challenge of Managing Continuous Change. Oak Brook: Council of Logistics Management, 1995.

GUNASEKARAN, A.; PATEL, C.; TIRTIROGLU, E. Performance measures and metrics in a supply chain environment. International Journal of Operations and Production Management, v. 21, n. 1-2, p. 71-87, 2001. http://dx.doi. org/10.1108/01443570110358468

HALLEY, A.; GUILHON, A. Logistics behaviour of small enterprises: performance, strategy and definition. International Journal of Physical Distribution and Logistics Management, v. 27, n. 8, p. 475-95, 1997. http://dx.doi. org/10.1108/09600039710182644

INSTITUTO BRASILEIRO DE GEOGRAFIA E ESTATÍSTICA IBGE. Índices Especiais - Bens de Capital. Disponível em: <http://www.ibge.gov.br>. Acesso em: 18 jan. 2008.

$\mathrm{KOH}, \mathrm{S}$. C. L. et al. The impact of supply chain management practices on performance of SMEs. Industrial Management and Data Systems, v. 107, n. 1, p. 103-24, 2007. http:// dx.doi.org/10.1108/02635570710719089
LAMBERT, D. M.; POHLEN, T. L. Supply chain metrics. International Journal of Logistics Management, v. 12, n. 1, p. 1-19, 2001. http://dx.doi.org/10.1108/09574090110806190

LAPIDE, L. MIT's SC2020 Project: The essence of excellence. Supply Chain Management Review, v. 10, n. 3, p. 18-24, 2006.

McDUFFIE, J. M. et al. Logistics transformed: the military enters a new age. Supply Chain Management Review, v. 5, n. 3, p. 92-100, 2001.

MOLLENKOPF, D.; DAPIRAN, G. P. World-class logistics: Australia and NewZealand. InternationalJournalofPhysicalDistribution and Logistics Management, v. 35, n. 1, p. 63-74, 2005. http://dx.doi.org/10.1108/09600030510577430

PESQUISA INDUSTRIAL DE INOVAÇÃO TECNOLÓGICA. Publicação PINTEC 2008. Disponível em: <http://www. pintec.ibge.gov.br>. Acesso em: 23 jun. 2011.

RAJEEV, N. Inventory management in small and medium enterprises: A study of machine tool enterprises in Bangalore. Management Research News, v. 31, n. 9, p.659-669, 2008. http://dx.doi.org/10.1108/01409170810898554

ROSENWEIG, E. D.; ROTH, A. V.; DEAN JUNIOR, J. W. The influence of an integration strategy on competitive capabilities and business performance: an exploratory study of consumer products manufacturers. Journal of Operations Management, v. 21, n. 4, p. 437-56, 2003. http://dx.doi.org/10.1016/S0272-6963(03)00037-8

SCHRAMM-KLEIN, H.; MORSCHETT, D. The relationship between marketing performance, logistics performance and company performance for retail companies. International Review of Retail, Distribution and Consumer Research, v. 16, n. 2, p. 277-96, 2006. http://dx.doi. org/10.1080/09593960600572399

SEBRAE. Aprenda com o Sebrae. Disponivel em: <http://www. sebrae.com.br>. Acesso em: 27 maio 2011.

SOUZA, J. H. A viabilidade do fomento tecnológico para a indústria de bens de capital em economias emergentes. Revista Spacios, v. 25, n. 1, p. 5-9, 2004.

TÖYLl, J. et al. Logistics and financial performance: an analysis of 424 Finnish small and medium-sized enterprises. International Journal of Physical Distribution and Logistics Management, v. 38, n. 1, p. 57-80, 2008. http://dx.doi. org/10.1108/09600030810857210

WEISE, M. R. 0 comportamento da indústria de bens de capital após o Plano Real. Revista FAE, v. 3, n. 3, p. 31-38, 2000.

\section{Abstract}

\section{A survey on SMEs logistics performance in the capital goods industry: a comparative analysis}

This paper presents the results of a survey research made on the logistics performance of small and medium enterprises (SMEs) from the capital goods industry. Performance was evaluated by means of the degree of concordance between companies and the logistics performance perspectives found in the literature. The results showed a great concordance of companies regarding such perspectives. After the comparative analysis, a substantial gap was found between the performance mean of SMEs and the logistics excellence reference. Further gaps were also identified in different segments, company size and capital source. Several practical and theoretical implications can be pointed out for these results, such as the need of partnerships among national and foreign companies and the systematization of best practices for this sector, which should be transferred to the SMEs that present lower logistics performance.

Keywords

Logistics. Logistical excellence. Logistics performance. Capital goods industry. 